\title{
Development of a New cryo-S(T)EM Technique Allowing Simultaneous STEM and SEM Imaging and its Application to Biological Samples
}

Jiro Usukura ( $\square$ usuura.jiro@b.mbox.nagoya-u.ac.jp )

Nagoya University

Akihiro Narita

Nagoya University

Tomoharu Matsumoto

Nagoya University

Eiji Usukura

Kyoto University

Takeshi Sunaoshi

Hitachi High-Tech Corporation

Syunya Watanabe

Hitachi High-Tech Corporation

Yusuke Tamba

Hitachi High-Tech Corporation

Yasuhira Nagakubo

Hitachi High-Tech Corporation

Takashi Mizuo

Hitachi High-Tech Corporation

Junzo Azuma

Hitachi High-Tech Corporation

Masako Osumi

Japan Women's University

Kazutaka Nimura

Hitachi High-Tech Corporation

Ryuichiro Tamochi

Hitachi High-Tech Corporation

Yoichi Ose

Hitachi High-Tech Corporation 
Keywords: Cryo-electron microscopy, SEM, STEM, Unroofing, Cytoskeleton, Actin filaments, Endoplasmic reticulum

Posted Date: May 26th, 2021

DOI: https://doi.org/10.21203/rs.3.rs-523739/v1

License: (1) This work is licensed under a Creative Commons Attribution 4.0 International License. Read Full License 


\section{Abstract}

A new type of cryo-electron microscopy (cryo-S(T)EM) technique made possible by installing a new cryotransfer holder and an anti-contamination trap on a scanning electron microscope (Hitachi SU9000) allowed simultaneous collection of both transmission (transmission electron microscopy, TEM) images and surface (scanning electron microscopy, SEM) images at $-180^{\circ} \mathrm{C}$. The ultimate temperatures of the cryo-transfer holder and the anti-contamination trap reached $-190^{\circ} \mathrm{C}$ and $-210^{\circ} \mathrm{C}$, respectively, by applying a liquid nitrogen slush. The TEM images obtained by the new cryo-S(T)EM method showed quality equal or superior to that of images obtained by conventional $100 \mathrm{kV} T E M$, although the resolution did not improve at $-180^{\circ} \mathrm{C}$ due to slight drifting of the sample stage. Cryo-S(T)EM also had the unexpected advantage of enabling observations of intracellular structures in thick frozen cells by accelerating the sublimation of ice surrounding the specimens. The spatial architecture of the cytoskeleton, poly-ribosome-chains, endoplasmic reticulum (ER), mitochondria, etc., became visible in thick frozen cells via sufficient (deep) sublimation of ice in combination with the unroofing method. In particular, it should be noted that the ER appeared as a wide and flat structure beneath the cell membrane while forming a large spatial network together with tubular ER.

\section{Introduction}

Cryo-transmission electron microscopy (cryo-TEM) has gradually become popular in the past decade, and several dedicated microscopes are currently sold commercially. In the past, independently sold cryotransfer holders were used in conjunction with general-purpose transmission electron microscopes to observe frozen samples because totally equipped microscopes dedicated to frozen samples were rarely available commercially. The major difference between these types of instruments lies in how the sample is brought into the electron microscope. Currently, frozen samples can be placed in a cassette-shaped holder and moved in and out with an autoloader integrated with a cryo-TEM instrument. Images can be acquired stably, but regulation of the specimen temperature for a short time is limited compared to that achieved when using a side entry cryo-transfer holder. Therefore, it is difficult for a cassette-type holder to reduce the thickness of the ice layer by quickly increasing the temperature in a vacuum during imaging. It is thus preferable to observe protein molecules embedded in thin layers of ice rather than to observe frozen cells and tissues embedded in large amounts of ice. Practically, to date, cryo-TEM has been used mainly for structural analysis of purified proteins and viruses using image processing (single-particle analysis), which itself is a means of structural analysis and has been used without necessarily combining it with cryo-TEM in the past. Since quick freezing retains the structure of proteins in a nearly native state, single-particle analysis of frozen proteins using cryo-TEM has become popular. In particular, cryo-TEM has recently achieved substantially improved resolution due to the incorporation of highsensitivity cameras. Its resolution approaches atomic resolution, similar to X-ray crystallography, despite dealing with dispersed protein particles ${ }^{1-5}$. This cryo-TEM method developed under such circumstances is naturally targeted to the structural analysis of protein molecules. The acceleration voltage of cryo-TEM recently reached as high as $300 \mathrm{kV}$. A cryo-TEM instrument equipped with a direct detection (high- 
sensitivity) camera is an extremely expensive microscope that is installed at the university level and shared across regions but not installed at the individual laboratory level. Not only purified proteins but also cells are preserved well in the nearly native state by quick freezing. Therefore, observation of cells with cryo-TEM is essential for a comprehensive understanding of the true intracellular structures. In fact, cryo-EM of cells has provided high-resolution structural information about cells and organelles such as desmosomes and nuclear lamina, which have never been observed by conventional methods ${ }^{6-23}$. In particular, when this technique is combined with tomography, the molecular structure of membranes and several types of filaments can be analysed three-dimensionally ${ }^{7-22}$. Atomic resolution is not always required in cell biology, but it is desirable to have a cryo-TEM instrument available in a laboratory so that frozen samples can be observed frequently, as is possible with conventional TEM. This is one of the reasons we developed a small cryo-electron microscope. The aim of our newly developed cryo-electron microscopy (cryo-EM) technique is to observe the fine structure of frozen cells with high contrast in the nearly native state. This new cryo-EM method was developed based on scanning electron microscopy (SEM) and utilizes an acceleration voltage of $30 \mathrm{kV}$, a detector for transmitted electrons and a field emission gun (FEG) that emits an extremely thin electron beam (0.4 nm in diameter) (Hitachi SU9000). Therefore, our new cryo-EM method enables simultaneous acquisition of a transmission image (TEM image) and a surface image (SEM image). The TEM image obtained by the new cryo-EM technique is a scanning transmission image in the strict sense, so the newly developed cryo-EM method was named cryo-S(T)EM.

\section{Results}

\section{Cryo-S(T)EM instrumentation}

Our developed cryo-S(T)EM instrument including of a newly developed cryo-transfer holder and an anticontamination trap was fully functional as a standard cryo-electron microscope (Fig. S1). Both the cryotransfer holder and the anti-contamination trap have a double pipe structure containing a pipe for venting gas, so each can be easily filled to the tip with liquid nitrogen. The specimen stage of the cryo-transfer holder could be cooled to $-190^{\circ} \mathrm{C}$ by a liquid nitrogen slush obtained by evaporation with a vacuum pump (Fig. S2). Similarly, the anti-contamination trap could be cooled to $-210^{\circ} \mathrm{C}$ by using a liquid nitrogen slush (Fig. S3). However, the temperature of the specimen stage used for observing samples was substantially dependent on the temperature of the anti-contamination trap. If the temperature of the anti-contamination trap was the same as or higher than the temperature of the specimen stage, the water molecules sublimated from the ice around the sample were not efficiently adsorbed onto the anti-contamination trap and were refrozen on the sample surface as frost, causing contamination. To prevent this issue, the temperature of the specimen stage had to be set to approximately $20^{\circ} \mathrm{C}$ above the temperature of the anti-contamination trap. Thus, the sample had to be observed above $-180^{\circ} \mathrm{C}$. The water adsorption capacity of the anti-contamination trap was very important, especially for observing cells embedded in large amounts of ice, which differs from the requirements for observing purified protein molecules. Therefore, the tip shape of the anti-contamination trap was designed to enclose the specimen stage of 
the cryo-transfer holder, as shown in Fig. 1. On the other hand, the specimen stage of the cryo-transfer holder slides into the holder to prevent frost formation after setting samples in a cryo-workstation (Fig. S4) and is pulled out during observation (Fig. 2). The cryo-S(T)EM instrument, which was developed based on a SEM instrument (Hitachi SU9000) with detectors for secondary electrons, transmission electrons and backscattered electrons, should, in principle, be able to capture three images simultaneously. However, since the irradiation dose was limited to $\sim 30$ electrons/ $\AA^{2}$ to protect the frozen cells from irradiation damage, backscattered electrons from the ice surface were too weak to generate an image. On the other hand, a secondary electron image (SEM image) was observed with little charge-up due to the electron conductivity of ice. The transmission images obtained by cryo-S(T)EM were formed by scanning an extremely thin electron beam emitted from a cold FEG (diameter of beam: $0.4 \mathrm{~nm}$, spherical aberration coefficient: $2 \mathrm{~mm}$, optimum opening angle: $11 \mathrm{mrad})^{24}$. The quality of the scanning transmission image (STEM image) was evaluated by image processing of the micrographs of negatively stained actin filaments. The raw STEM image (Fig. 3A) clearly showed fine details with high contrast. The molecular model reconstructed by image processing also showed higher resolution than the model reconstructed from images obtained by conventional TEM (compare B with $C$ in Fig. 3). These results showed that the scanning transmission electron microscopy (STEM) mechanism within the abovedescribed cryo-S(T)EM method (or Hitachi SU9000 which is the basic machine for development) had the ability to display STEM images of biological samples equal to or better than conventional TEM images. Unfortunately, however, at $-180^{\circ} \mathrm{C}$, the resolution obtained at room temperature was not achieved due to drifting of the specimen stage. Therefore, high-resolution analysis will require the fabrication of a new drift-free cryo-transfer holder.

\section{Application of cryo-S(T)EM to the analysis of cell structures}

Since quick freezing can be used to preserve not only purified proteins but also the fine structure of cells in a nearly native state, cells also need to be observed in the frozen state to understand their real fine structure. Of course, the slight drift of the specimen stage mentioned above makes high-resolution cell observation difficult, but cryo-S(T)EM combined with the unroofing method can yield valuable results for thick cell structures even at low resolution. Cultured cells are extremely thick, reaching a few microns, and are frozen together with a large amount of water compared to purified proteins. Therefore, it is necessary to reduce the thickness of frozen samples enough to allow electrons to pass through. In previous studies, frozen samples were thinned by using a cryo-ultramicrotome (CEMOVIS) ${ }^{7}$ or focused ion beam (FIB) ${ }^{19-}$ 22. In this study, unroofing $23,25,26$ was used instead. Initially, unroofing is a unique method for exposing the cytoplasmic surface of the ventral cell membrane to observe clathrin coats, caveolae, etc., in freezeetching replica EM ${ }^{27,28}$. In practice, however, all cells are not unroofed in the same way. Unroofed cells vary in thickness and cytoplasmic contents, ranging from fully unroofed cells to non-unroofed cells. The observations of various unroofed cells by cryo-S(T)EM are described below, and the cells are classified into fully unroofed cells, partially unroofed cells and small (micro-) unroofed cells (see Fig. S5 for better understanding). In many cases, frozen cells were embedded in enough ice to prevent the transmission of electrons. To reduce the thickness of the ice layer, the temperature of the specimen stage was first increased to $-100^{\circ} \mathrm{C}$ to accelerate ice sublimation, and thereafter, the temperature was decreased to 
$-180^{\circ} \mathrm{C}$ again for observation. This ability to easily adjust the ice thickness by raising or lowering the temperature of the specimen stage is another advantage of this cryo-S(T)EM technique that is very useful for observing various unroofed cells. In fully unroofed cells (cells with little cytoplasm), the cytoplasmic surface (inner surface) of the ventral cell membrane was exposed thoroughly, and clathrin coats, cortical actin filaments, and microtubules were found in close contact with the surface (Fig. 4). Actin filaments and microtubules grew without fragmentation or branching while bending gently. Partially unroofed cells reached a few microns in thickness due to residual organelles and cytoplasm, although most of the cell membrane was removed. In general, it is difficult for electrons to pass through such a thick sample to generate an image. However, surprisingly, sufficient (deep) sublimation of ice exposed intracellular fine structures, regardless of the degree of unroofing. The reason the structures became visible after ice sublimation even though the cell thickness did not change will be explained below (DISCUSSION section). Regardless, the intracellular structures became clearer with increasing duration of ice sublimation. Figure 5 shows partially unroofed cells whose cytoplasm and water were frozen to an appropriate extent. Actin filaments, ribosomes, endoplasmic reticulum (ER), etc., appeared after slight ice sublimation. These cells are completely embedded in ice, and thus, the SEM image shows a flat surface. Cells unroofed to this extent provide the most information about structures. Figure 5B shows a high-magnification micrograph of another part of the same cell shown in Fig. 5A. The ER, ribosomal chain, microtubules, and actin filaments clearly overlap with each other. On the other hand, in the case of another partially unroofed cell with considerable thickness (Fig. 6), ice sublimation for $20-30$ minutes at $-100^{\circ} \mathrm{C}$ was required for TEM images to be obtained. Figure 6 shows the nucleus, mitochondria, ER and filaments. Sufficient (deep) sublimation also revealed organelles embedded in ice in an SEM image (Fig. 6 right image). Thus, simultaneous measurement of SEM images was useful for investigating the freeze-drying state. Notably, in this study, the intracellular fine structure of micro-unroofed cells, which is similar to the whole cell, appeared after deep sublimation of ice even during $30 \mathrm{kV}$ cryo-S(T)EM. In fact, as shown in Fig. 7, since the SEM image was flat even after sufficient sublimation, it was considered that most parts of these cells are covered with a cell membrane. Nevertheless, the mitochondria, ER, and cytoskeleton became visible upon deep sublimation. Many flat ERs were located beneath the apical cell membrane (Fig. 7) and seemed to be interconnected with each other to form a network. These ER networks were also well preserved in mitotic telophase cells, as shown in Fig. 8. These structures had never before been observed in thin sections of cells. The ability to observe thick cells in combination with deep sublimation of ice is one of the advantages of this developed cryo-S(T)EM technique.

\section{Discussion}

We aimed to develop a compact cryo-EM instrument based on the $30 \mathrm{kV}$ SEM with a STEM detector. This is the first cryo-EM that is able to display both a transmission image (STEM image) and a secondary electron image (SEM image, surface image) at the same time. Effective simultaneous capture of these images of unfixed cells seems to be possible only in the frozen state. The high electrical conductivity of both frozen cells and grids covered with carbon-coated film allows SEM images to be obtained while suppressing electron charge along the sample surface. Therefore, simultaneous STEM and SEM imaging 
became possible without any pre-treatment, such as metal decoration. However, electrons back-scattered from the frozen sample surface were too weak to generate images because the total number of irradiated electrons was reduced to prevent irradiation damage.

STEM images obtained by $30 \mathrm{kV}$ cryo-S(T)EM were equal or superior in quality to images obtained by conventional $100 \mathrm{kV}$ TEM at room temperature in this study. However, the image quality decreased at $-180^{\circ} \mathrm{C}$ due to drifting of the specimen stage. Structural analysis of proteins with the highest resolution of cryo-S(T)EM using image processing at $-180^{\circ} \mathrm{C}$ will be a challenge until fabrication of a new drift-free cryo-transfer holder. Issues of image quality in STEM are closely related to scan speed and detector sensitivity, in addition to microscope-specific optics issues. The scanning speed of SEM has hardly changed in the last 50 years, and the capture speed in image acquisition mode is approximately 20 to 30 s per frame. In this study, 2560×1920 pixel images were recorded in $32 \mathrm{~s} /$ frame, and 1280×960 pixel images were recorded in $16 \mathrm{~s} /$ frame. Capture speed is also closely related to detector sensitivity. The higher the sensitivity, the faster the capture speed is. Therefore, it will be necessary to improve the sensitivity of the detector and the scanning speed in the future. If the detection sensitivity and scanning speed are sufficiently high and the image can be captured at a speed greater than the sample drift, the drift problem will cease.

The new cryo-S(T)EM method employed unroofing for sample preparation and yielded several new findings on intracellular structures in the nearly native state. Samples have to be frozen immediately after unroofing to maintain their native structures. As mentioned above, the amount of remaining cytoplasm varied among unroofed cells from part of the cell membrane to a whole cell. The total thickness of microunroofed or slightly unroofed cells reached a few microns because many organelles remained in the cytoplasm. The samples were not thin enough to allow electrons to pass through at an acceleration voltage of $30 \mathrm{kV}$. This fact raises the question of why the structure can be observed simply by sublimating a large amount of ice. Even though the cells appeared similar to normal cells, they were either slightly unroofed or had micron-sized holes, as described above. In this case, the cell fluid (cell sap) was gradually diluted by influx of an external buffer and concomitant outflow of the cell fluid through partially broken areas or micron-sized holes in the cell membrane. Although the normal frozen cell fluid is hardly sublimated even under high vacuum, the frozen cell fluid diluted sufficiently with buffer was gradually sublimated by increasing the specimen temperature to $-100^{\circ} \mathrm{C}$ under high vacuum $\left(5 \times 10^{-6} \mathrm{~Pa}\right)$ for 30 minutes or more. Deep sublimation of ice caused a significant decrease in frozen cell fluid, allowed electron beams to pass through and generate an image, thus explaining why the structure of thick cells appeared after deep sublimation. Prolonged sublimation of ice surrounding frozen cells has previously been considered to cause some artefacts. Therefore, increasing the specimen temperature to accelerate the sublimation of ice has not been attempted in the past. However, no artefacts have yet been detected on images taken at 40,000 magnification. When ice is sublimated in a vacuum, the constituent minerals in the buffer should precipitate on the surface of the membrane and filaments. However, the amount of precipitation is too small to be detected in an image at 50,000 or less. When the sample is completely dried, more components of the buffer precipitate on the exposed structure and may be observed as 
contaminants. However, the fine structures collapsed upon drying completely in previous experiments. The fine structure of unroofed cells should be supported by an ice layer. When the total thickness of the ice containing frozen cell fluid was reduced to $200 \mathrm{~nm}$ or less by sublimation in vacuo, the electron beam was sufficiently transmitted to generate an image. The organelles that were completely exposed from the ice layer also appeared to be covered with a very small amount of ice. This ices appears to maintain the structure of unfixed cells. Therefore, sublimation of ice should be minimized.

\section{Methods}

\section{Construction of a new cryo-scanning transmission electron microscope (cryo-S(T)EM)}

A scanning electron microscope (Hitachi SU9000; accelerating voltage, $30 \mathrm{kV}$ ) equipped with a detector for transmitted electrons, detectors for secondary electrons and a detector for back-scattered electrons was used as the base machine of the development. The cryo-S(T)EM instrument consisted of the above SEM (Hitachi SU9000) with a new anti-contamination trap (Fig. 1) and a new cantilever-type cryo-transfer holder (Fig. 2). Anti-contamination traps are very important to prevent refreezing of sublimated water molecules as frost on the specimen surface and are always installed in cryo-S(T)EM instruments. After mounting the frozen cells onto the specimen stage of the cryo-transfer holder in liquid nitrogen with the assistance of a newly developed cryo-workstation (Fig. S3), the specimen stage was slid into the cryotransfer holder to prevent frost (Fig. 2). Then, the cryo-transfer holder was placed in the specimen chamber of the cryo-S(T)EM instrument. Such sample loading must be performed for each observation. Liquid nitrogen in the Dewar of the cryo-transfer holder and anti-contamination holder was evaporated as necessary by means of a vacuum pump to generate nitrogen slush. Evaporation was stopped before observation to prevent vibration from the vacuum pump. The specimen temperature was maintained for 40 minutes after evaporation stopped. Samples were observed during this time at $-180{ }^{\circ} \mathrm{C}$ while monitoring the temperature. If the sample temperature became unstable and rose during observation, evaporation was started again for approximately 5 minutes to restore the nitrogen slush. Then, observation was resumed.

\section{Sample preparation for cryo-EM}

Normal rat kidney (NRK) cells were cultured on C-flat gold mesh grids (\#200 multi-hole) or molybdenum mesh grids (\#200) covered with carbon-coated Formvar (polyvinyl formal) for 1-2 days in a $\mathrm{CO}_{2}$ incubator. The culture medium was DMEM (Sigma-Aldrich Co., St. Louis, MO, USA) supplemented with 10 $\%$ bovine serum. The sonication unroofing method was used to observe the membrane cytoskeleton in cells. Unroofing also helped prepare cells thin enough for electron beams to pass through. Cells cultured on the above mesh grids were sequentially washed with Ringer's solution consisting of $155 \mathrm{mM} \mathrm{NaCl}, 3$ $\mathrm{mM} \mathrm{KCl}, 2 \mathrm{mM} \mathrm{CaCl}_{2}, 1 \mathrm{mM} \mathrm{MgCl}_{2}, 3 \mathrm{mM} \mathrm{NaH}_{2} \mathrm{PO}_{4}$, and $10 \mathrm{mM}$ glucose in $5 \mathrm{mM}$ HEPES buffer (pH 7.4), followed by $\mathrm{Ca}^{2+}$-free Ringer's solution (lacking $\mathrm{CaCl}_{2}$ ). The cells were soaked for approximately $10 \mathrm{~s}$ in a 
polylysine solution (Mw 30,000-50,000; Sigma-Aldrich, St. Louis, MO, USA; $0.5 \mathrm{mg} / \mathrm{ml}$ dissolved in $\mathrm{Ca}^{2+}$ free Ringer's solution) and then washed three times for a few seconds each in $\mathrm{KHMgE}$ buffer consisting of $30 \mathrm{mM}$ HEPES (pH 7.4), $70 \mathrm{mM} \mathrm{KCl}, 3 \mathrm{mM} \mathrm{MgCl}_{2}$, and $1 \mathrm{mM} \mathrm{EGTA}$. Immediately after washing, the cells were unroofed by gentle sonication $(0.5 \mathrm{~W}, 27 \mathrm{kHz})$ in isotonic unroofing buffer consisting of $30 \mathrm{mM}$ HEPES (pH 7.4), 70 mM KCl, 3 mM MgCl, 1 mM EGTA, 1 mM DTT and Pefabloc SC (Roche Diagnostics Gmbh, Mannheim Germany) as a protease inhibitor. The unroofed cells were washed briefly in the same fresh buffer and used for subsequent experiments. See previous papers for details 26,27 .

\section{Quick freezing}

Unroofed samples were immediately frozen by using a Leica EM-GP quick freezer. Excess water surrounding the unroofed cells was automatically absorbed by filter paper for $5-6 \mathrm{~s}$ from only the grid side in a Leica EM-GP quick freezer. Then, the samples were automatically plunged into liquid ethane cooled at $-185^{\circ} \mathrm{C}$ with liquid nitrogen. Frozen samples were stored temporarily in liquid nitrogen for subsequent experiments.

\section{Sublimation of ice}

Cells were often embedded in a large amount of ice even after water absorption during Leica EM-GP. Cells embedded in a relatively thin layer of ice were selected for observation, but sometimes further sublimation of ice was required to reduce the total thickness. Sublimation of ice was performed by increasing the temperature to $-100^{\circ} \mathrm{C}$ under high vacuum $\left(5 \times 10^{-6} \mathrm{~Pa}\right)$ during cryo-S(T)EM. The thickness of the ice was estimated from a low-magnification SEM image (mesh image). Upon reaching an appropriate thickness, the temperature was lowered again to $-180^{\circ} \mathrm{C}$ for observation.

\section{Image processing}

The image quality of negatively stained actin filaments achieved by Cryo-S(T)EM was evaluated by image processing with EOS software ${ }^{31}$. Details of actin purification and single-particle analysis of actin filaments using spiral symmetry were described in previous

papers $^{30,32,33}$.

\section{Declarations}

\section{Funding}

This work was mainly supported by the Development of Advanced Measurement and Analysis Systems (AMED-SENTAN) [\#18hm0102003s0107 to J.U.] program funded by the Japan Agency for Medical Research and Development and partly supported by a Grant-in-Aid for Scientific Research [\#20K06586 to J.U. and \#19K06583 to E.U.] from JSPS. 


\section{Author contributions}

All authors have been involved in the development of a new cryo-S(T)EM technique in their respective areas of expertise. J.U. and E.U. prepared the unroofed frozen cells and improved the protocols of unroofing. A.N. and T.M. were in charge of image processing of actin filaments. J.U. wrote the manuscript with contributions from all the authors.

\section{Conflict of interests}

The authors declare no conflicts of interest.

\section{References}

1. Henderson, R. \& McMullan, G. Problems in obtaining perfect images by single-particle electron cryomicroscopy of biological structures in amorphous ice Microscopy62, 43-50 (2013).

2. Frank J Generalized single-particle cryo-EM - a historical perspective Microscopy65, 3-8 (2016).

3. Kato, T. et al. CryoTEM with a Cold Field Emission Gun That Moves Structural Biology into a New Stage Microsc. Microanal.25, 998-999 (2019).

4. Danev, R., Yanagisawa, H. \& Kikkawa, M. Cryo-Electron Microscopy Methodology: Current Aspects and Future Directions. Trends Biochem. Sci.10, 837-848 (2019).

5. Xie, Q., Yoshioka, C.K. \& Chapman, M.S. Adeno-Associated Virus (AAV-DJ)-Cryo-EM Structure at 1.56 Angstroms Resolution. Viruses12, 1194; 10.3390/v12101194 (2020).

6. Resch, G. P., Goldie, K. N., Krebs, A., Hoenger, A. \& Small, J. V. Visualization of the actin cytoskeleton by cryo-electron microscopy. Cell Sci.115, 1877-1882 (2002).

7. Baumeister, W. Electron tomography: toward visualizing the molecular organization of the cytoplasm. Curr. Opin. Struct. Biol 12, 679-684 (2002).

8. Medalia, 0 . et al. Macromolecular architecture in eukaryotic cells visualized by cryoelectron tomography. Science298, 1209-1213 (2002).

9. Al-amoudi, A. et al. Cryo-electron microscopy of vitreous sections. EMBO J.23, 3583-3588 (2004).

10. Kurner, J., Medalia, O., Linaroudis, A. A. \& Baumeister, W. New insights into the structural organization of eukaryotic and prokaryotic cytoskeletons using cryo-electron tomography. Exp. Cell Res.301, 3842 (2004).

11. Kurner, J., Frangakis, A. S. \& Baumeister W Cryoelectron tomography reveals the cytoskeletal structure of Spiroplasma melliferum. Science307, 436-438 (2005).

12. Lucic, V., Yang, T., Schweikert, G., Forster, F. \& Baumeister, W. Morphological characterization of molecular complexes present in the synaptic cleft. Structure13, 423-434 (2005)

13. Marko M. Hsieh C. Schalek R. Frank J. \& Mannella C. Focused-ion-beam thinning of frozen-hydrated biological specimens for cryo-electron microscopy. Nat. Methods.4, 215-217 (2007). 
14. Patla, I. et al. Dissecting the molecular architecture of integrin adhesion sites by cryo-electron tomography. Nat. Cell Biol.12, 909-915 (2010)

15. Elad, N., Abramovitch, S., Sabanay, H. \& Medalia O Microtubule organization in the final stages of cytokinesis as revealed by cryo-electron tomography. J. Cell Sci. 124, 207-215 (2010).

16. Bokstad, M., Sabanay, H., Dahan, I., Geiger, B. \& Medalia, O. Reconstructing adhesion structures in tissues by cryoelectron tomography of vitrified frozen sections. J. Struct. Biol.178, 76-83 (2012).

17. Harapin, J., Elbauer, M. \& Medalia, O. Structural analysis of supramolecular assemblies by cryoelectron tomography. Structure21, 1522-1530 (2013)

18. Dubrovsky, A., Sorrentino, S., Harapin, J., Sapra, K. T. \& Medalia, O. Developments in cryo-electron tomography for in situ structural analysis. Arch. Biochem. Biophys.581, 78-85 (2015)

19. Tamir, A. et al. The macromolecular architecture of platelet derived microparticles. Struct. Biol. 193, 181-187 (2015)

20. Mahamid, J. et al. Visualizing the molecular sociology at the HeLa cell nuclear periphery. Cell Nuclear 351, 969-972 (2016).

21. Weber, M.S., Wojtynek, M. \& Medalia, O. Cellular and structural studies of eukaryotic cells by cryoelectron tomography. Cells8, 57 1-18 (2019)

22. Kronenberg-Tenga, R. et al. A lamin A/C variant causing striated muscle disease provides insights into filament organization J. Cell Sci.134, (6) jcs256156 (2021).

23. Makihara, M. et al. A new approach for the direct visualization of the membrane cytoskeleton in cryoelectron microscopy: a comparative study with freeze-etching electron microscopy. Microscopy65, 488-498 (2016).

24. Konno, M., Ogashiwa, T., Sunaoshi, T., Orai, Y. \& Sato, M. Lattice imaging at an accelerating voltage of $30 \mathrm{kV}$ using an in-lens type cold field-emission scanning electron microscope. Ultramicroscopy 145 , 28-35 (2014).

25. Oda, T., Iwasa, M., Aihara, T., Maeda, Y. \& Narita, A. The nature of the globular- to fibrous-actin transition Nature457 441-445 (2009)

26. Usukura, E. Narita, A. Yagi, A. Ito, S. \& Usukura, J. An unroofing method to observe the cytoskeleton directly at molecular resolution using atomic force microscopy. Sci. Rep.6, 27472; 10.1038/srep27472 (2016).

27. Morone, N., Usukura, E., Narita, A. \& Usukura, J. Improved unroofing protocols for cryo-electron microscopy, atomic force microscopy and freeze-etching electron microscopy and the associated mechanisms. Microscopy69 350-359 (2020).

28. Heuser, J. The production of 'cell cortices' for light and electron microscopy. Traffic1: 545-552 (2000)

29. Morone, N. et al. Three-dimensional reconstruction of the membrane skeleton at the plasma membrane interface by electron tomography. Cell Biol.174, 851-862 (2006). 
30. Spudich, J.A. \& Watt, S. The regulation of rabbit skeletal muscle contraction. I. Biochemical studies of the interaction of the tropomyosin-troponin complex with actin and the proteolytic fragments of myosin. J. Biol. Chem. 246, 4866-4871(1971).

31. Yasunaga, T. \& Wakabayashi, T.Extensible and Object-Oriented System Eos Supplies a New Environment for Image Analysis of Electron Micrographs of Macromolecules.

J. Struct. Biol.116, 155-160 (1996).

32. Narita, A. et al. $\mathrm{Ca}^{2+}$-induced switching of troponin and tropomyosin on actin filaments as revealed by electron cryo-microscopy. J. Mol. Biol. 308, 241-261 (2001).

33. Popp, D., et al. Molecular structure of the ParM polymer and the mechanism leading to its nucleotidedriven dynamic instability EMBO J. 27, 570-579 (2008).

\section{Figures}

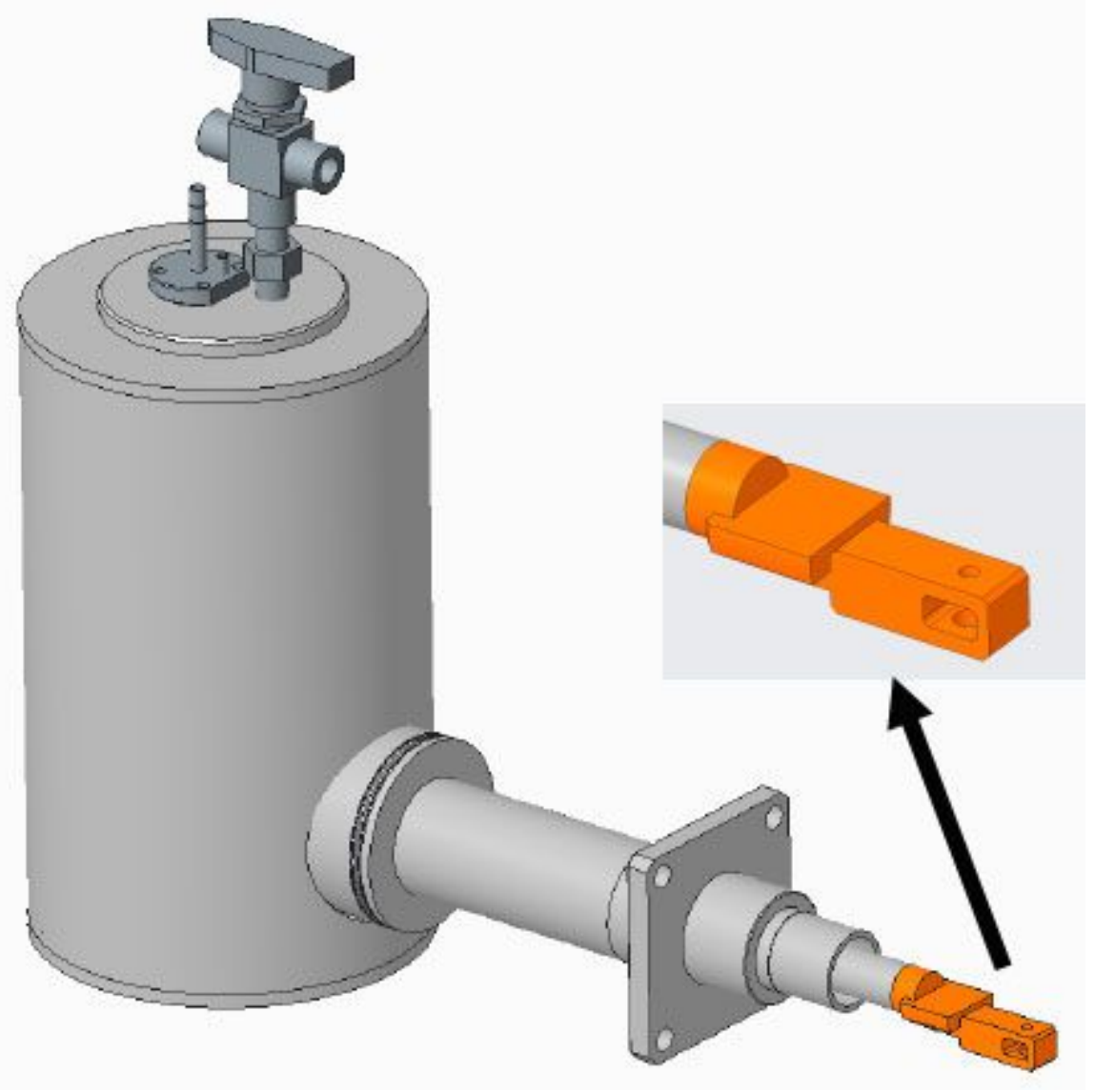

\section{Figure 1}

Illustration of an anti-contamination trap. The inset shows an enlarged view of the tip of the anticontamination trap. 

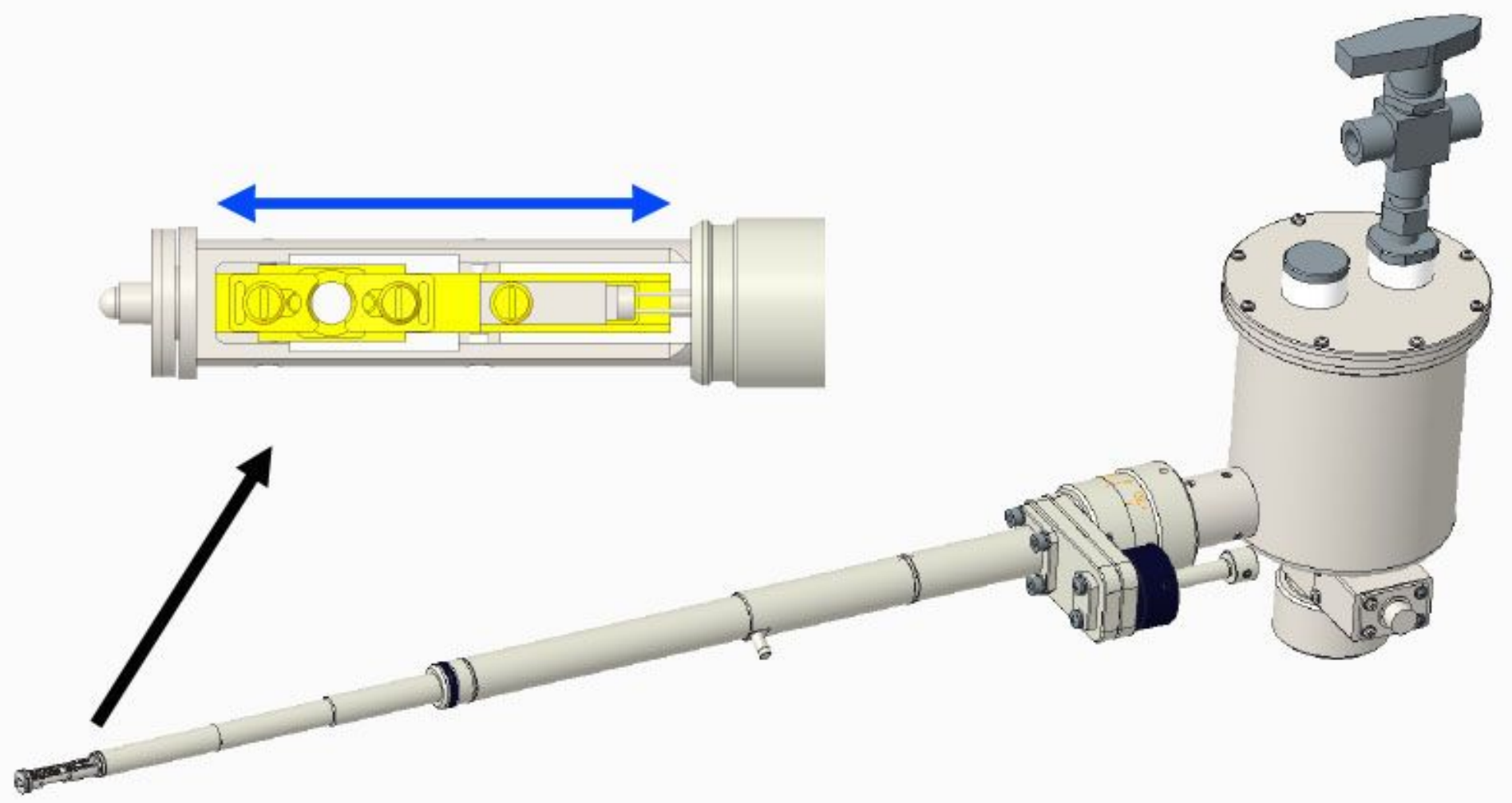

Figure 2

Illustration of a cryo-transfer holder. The inset shows an enlarged view of the specimen stage at the tip of the cryo-transfer holder. This specimen stage slides into the arm of the cryo-transfer holder to prevent frost formation during transfer it into the specimen chamber of the cryo-S(T)EM
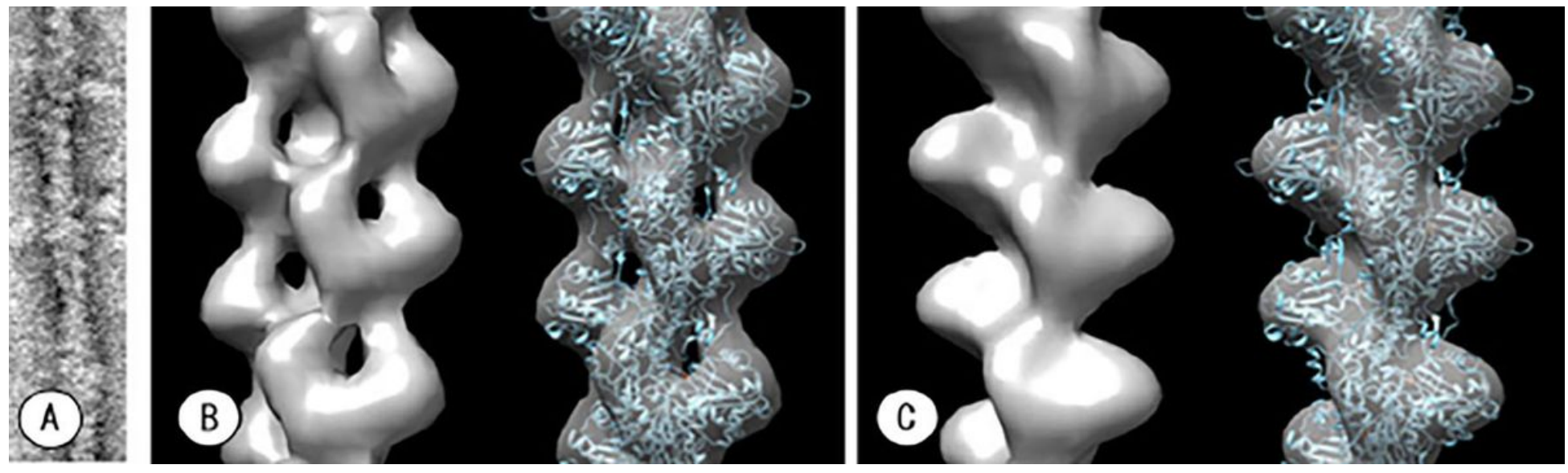

\section{Figure 3}

Comparison of two molecular models reconstructed by image processing of each actin filament image captured by $30 \mathrm{kV}$ cryo-S(T)EM and $100 \mathrm{kV}$ conventional TEM. A: One raw image of negatively stained actin filaments obtained by the developed $30 \mathrm{kV}$ cryo-S(T)EM technique. B: Molecular model of actin filaments reconstructed by image processing from 24 filaments images with the total length of 9 micrometer, containing approximately 3000 actin monomers images, obtained by cryo-S(T)EM; the molecular 
mapping derived by X-ray diffraction was superimposed to determine how well the reconstructed model matches the actual molecular structure25. C: Molecular model of actin filaments reconstructed by image processing from 19 filaments images with the total length of 9 micro-meter obtained by conventional TEM; the molecular mapping same as in B was also superimposed25. By comparing Models B and C, it is clear that Model $\mathrm{B}$ is more consistent with the actual actin molecular mapping.
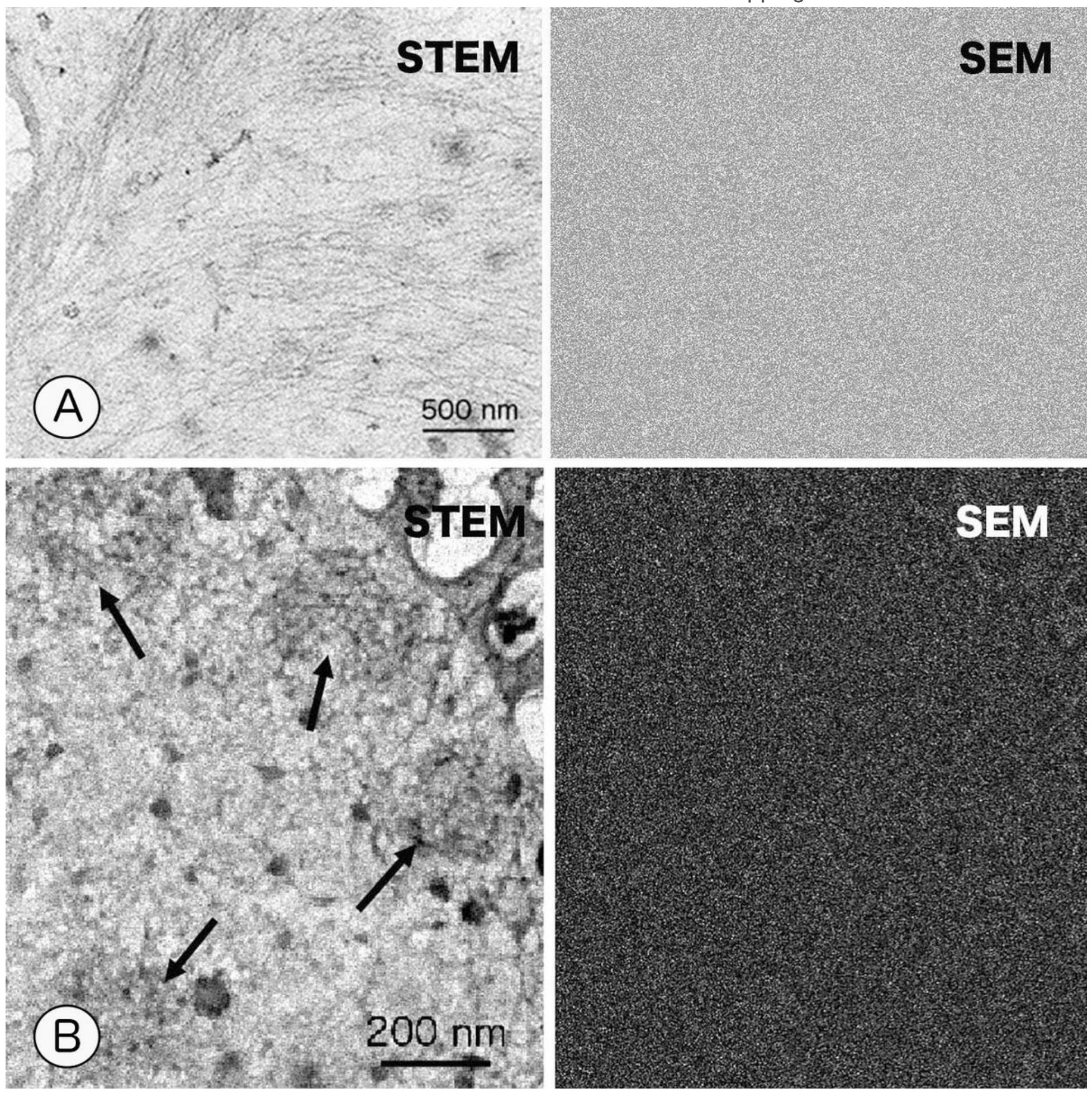

Figure 4 
Simultaneous imaging by cryo-STEM and cryo-SEM showing fully unroofed cells. A: Cortical actin filaments attached on the cytoplasmic (inside) surface of a cell membrane. B: Clathrin coats (arrows) were observed on the inner surface of the cell membrane in fully unroofed cells. The thickness of fully unroofed cells is thin enough for electrons to pass through. The SEM image appears flat because the unroofed cells are completely embedded in ice. In C, however, since ice sublimation is somewhat accelerated, the structure in SEM mode was observed.
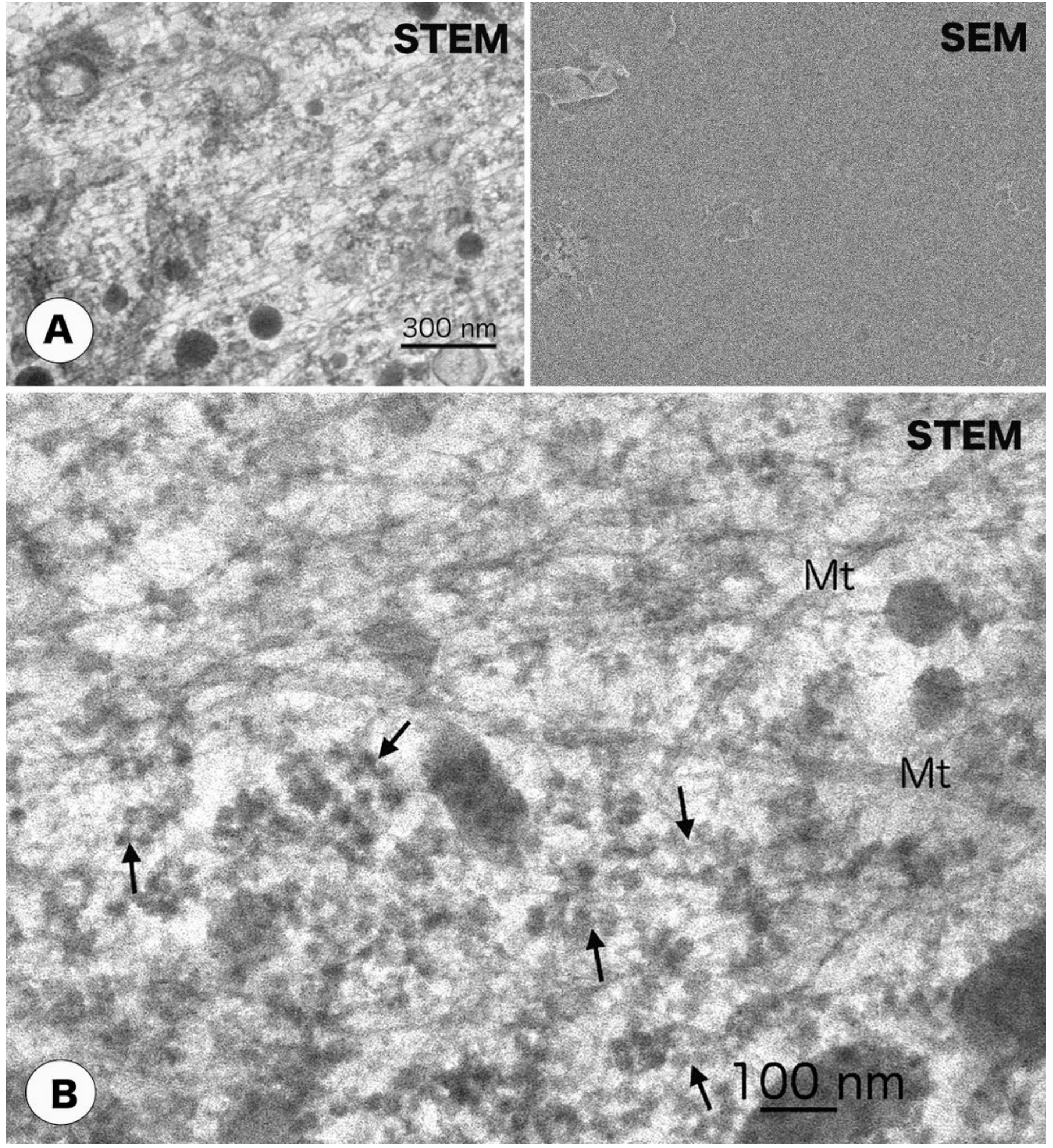

Figure 5 
Cryo-electron micrographs of partially unroofed cells. A: Simultaneous imaging by cryo-STEM and cryoSEM of cytoskeletons, ribosomes and endoplasmic reticulum. In partially unroofed cells, the cell membrane was removed, but many organelles remained intact. The total thickness of the cell was reduced so much by unroofing that STEM images could be observed with slight sublimation of ice. The unroofed cell seems to be completely embedded in ice, as shown by the flatness of the SEM image. B: Magnified cryo-STEM micrograph of the other part of the same sample shown in Figure A. Polyribosomal-chains (arrows) and microtubules (Mt) are clearly observed. Ribosomes are often interconnected by extremely thin threads to form a ribosome chain. Since these structures are observed to overlap with other filaments and membrane structures, this unroofed cell appears to be quite thick.
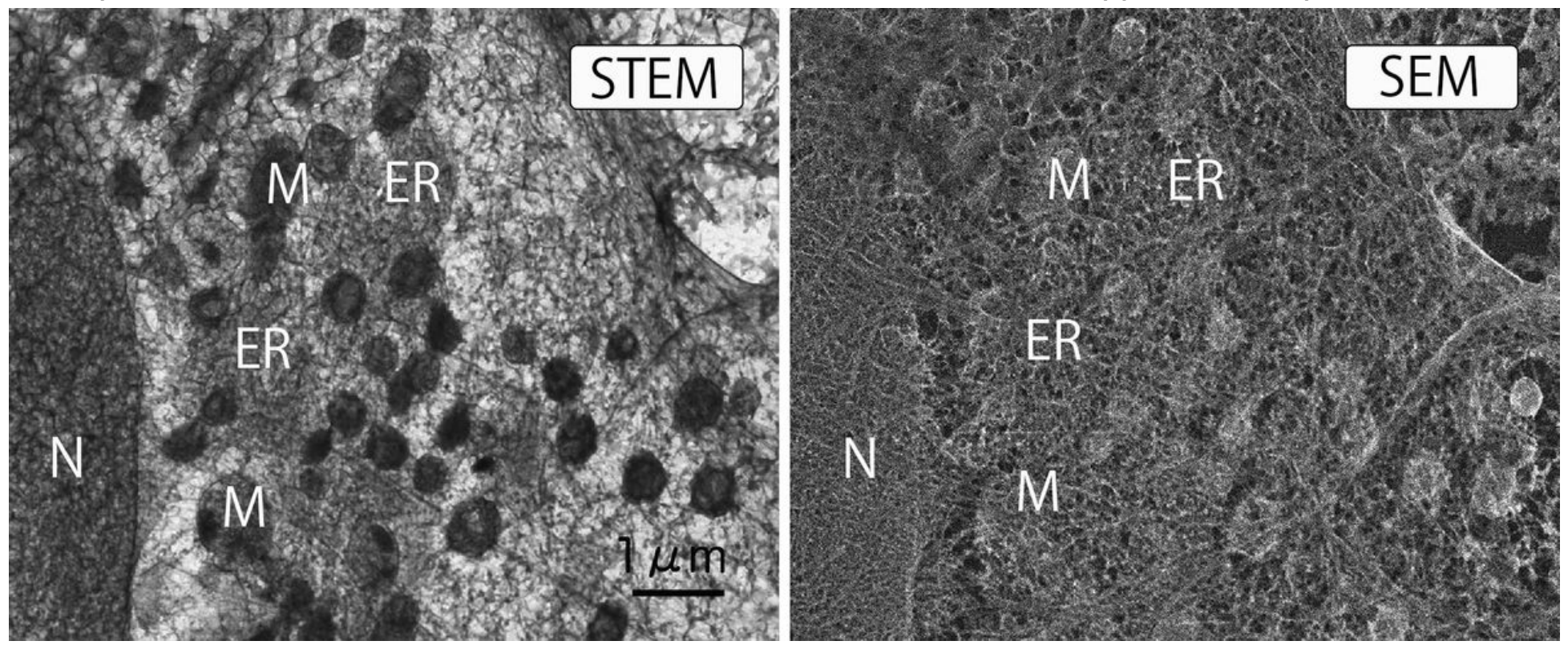

\section{Figure 6}

Simultaneous imaging by cryo-STEM and cryo-SEM showing a partially unroofed cell. Many organelles remain in spite of complete ablation of the cell membrane. Deep sublimation made ice layer thin for electrons to pass through total thickness of cell, and thereby STEM images became visible. At the same time, the undulation in the cytoplasm could be observed in the SEM image. N; nucleus, M; mitochondria, ER; endoplasmic reticulum. 

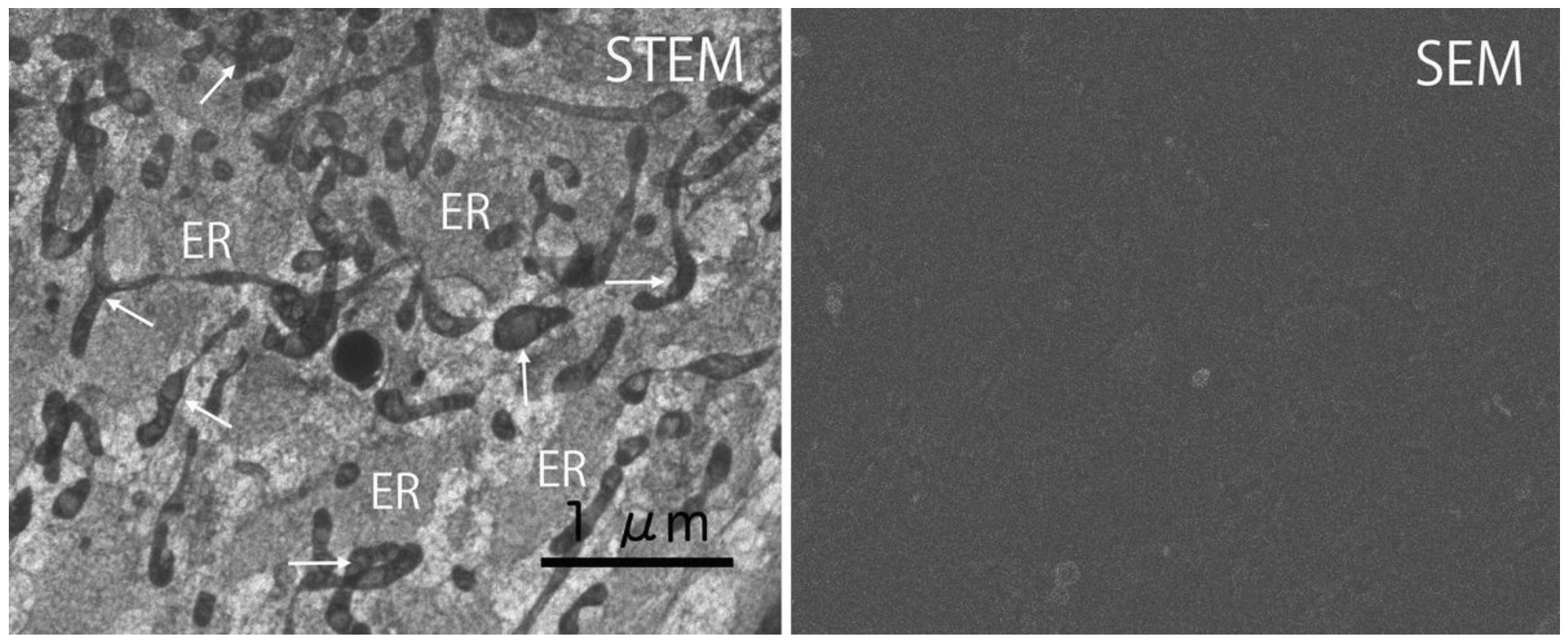

Figure 7

Simultaneous imaging by cryo-STEM and cryo-SEM showing a micro-unroofed cell with many organelles. A micro-unroofed cell is similar to a normal cell in appearance, but a very small area of the cell membrane is torn off or very tiny holes have been opened. The SEM image was not observed even after ice sublimation for one hour or more because most areas of the cell were covered by the cell membrane. As seen in this micrograph, STEM revealed the spatial architecture of several organelles and filaments as in high-resolution light microscopy. In this micrograph, a large ER network was observed in the vicinity of the cell membrane. Mitochondria were also observed, sometimes branching or overlapping with the ER. 


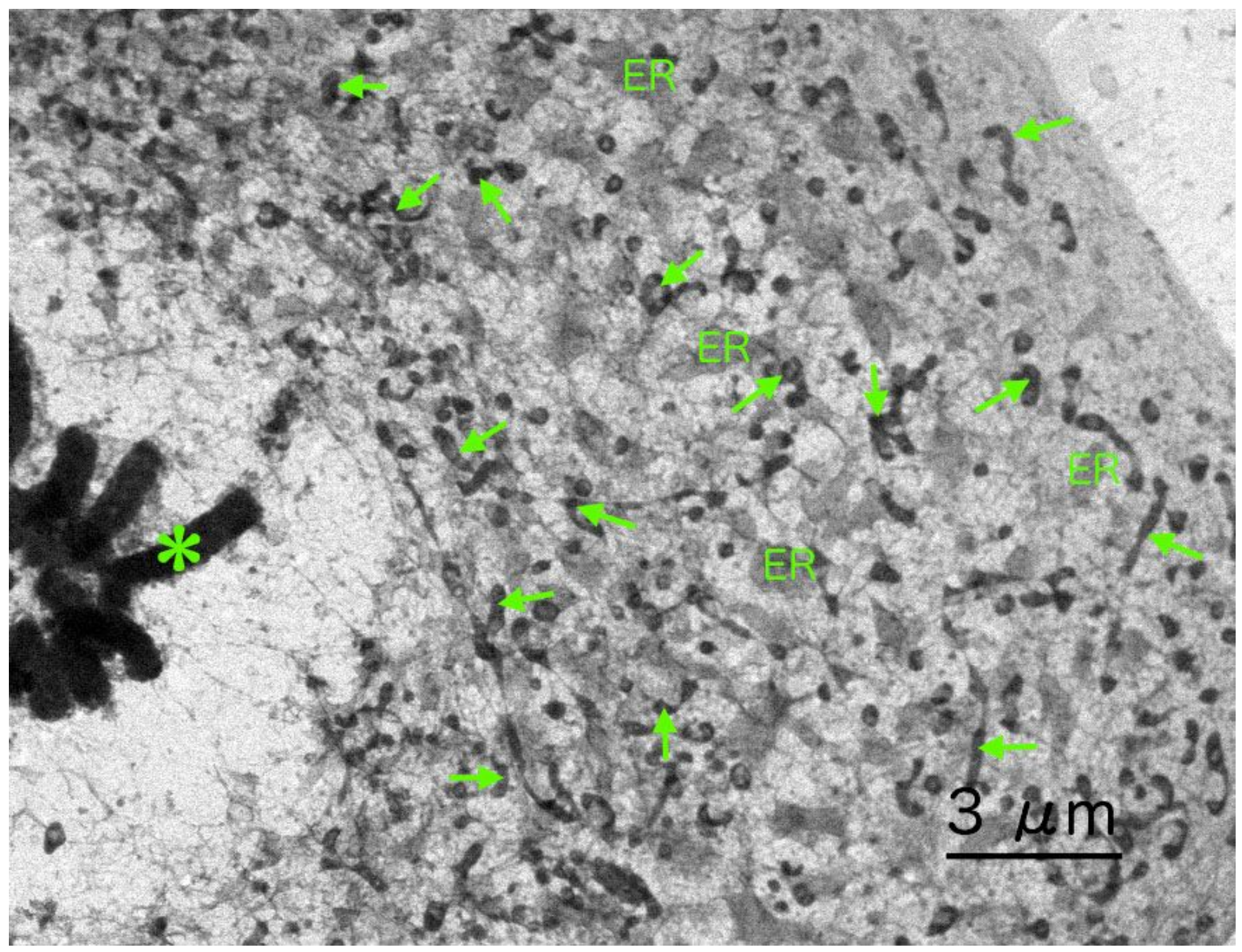

Figure 8

Cryo-electron micrograph of micro-unroofed cells in mitotic telophase. The arrows indicate mitochondria. ER indicates the endoplasmic reticulum. The asterisk shows chromosomes in telophase. Large networks of ER were preserved well even in mitotic phase despite the disappearance of the nuclear envelope.

\section{Supplementary Files}

This is a list of supplementary files associated with this preprint. Click to download.

- SUPPLEMENTS.pdf 